\title{
Detecting sound hard cracks in isotropic inhomogeneities
}

Lorenzo Audibert ${ }^{1,2}$, Lucas Chesnel², Houssem Haddar $^{2}$, and Kevish Napal ${ }^{2}$

\begin{abstract}
We consider the problem of detecting the presence of sound-hard cracks in a non homogeneous reference medium from the measurement of multi-static far field data. First, we provide a factorization of the far field operator in order to implement the Generalized Linear Sampling Method (GLSM). The justification of the analysis is also based on the study of a special interior transmission problem. This technique allows us to recover the support of the inhomogeneity of the medium but fails to locate cracks. In a second step, we consider a medium with a multiply connected inhomogeneity assuming that we know the far field data at one given frequency both before and after the appearance of cracks. Using the Differential Linear Sampling Method (DLSM), we explain how to identify the component(s) of the inhomogeneity where cracks have emerged. The theoretical justification of the procedure relies on the comparison of the solutions of the corresponding interior transmission problems without and with cracks. Finally we illustrate the GLSM and the DLSM providing numerical results in 2D. In particular, we show that our method is reliable for different scenarios simulating the appearance of cracks between two measurements campaigns.
\end{abstract}

Key words: inverse scattering, sampling methods, sound-hard cracks.

\section{Introduction}

This work is a contribution to sampling methods in inverse scattering theory when the issue is to determine the shape of an unknown inclusion from fixed frequency multi-static data. More precisely we extend the Generalized Linear Sampling Method (GLSM) and the Differential Linear Sampling Method (DLSM) [2, 3] to inhomo-

\footnotetext{
${ }^{1}$ Department PRISME, EDF R\&D, 6 quai Watier, 78401, Chatou CEDEX, France.

2 INRIA/Centre de mathématiques appliquées, École Polytechnique, Université Paris-Saclay, Route de Saclay, 91128 Palaiseau, France. e-mail: lorenzo.audibert@edf.fr, lucas.chesnel@inria.fr, houssem.haddareinria.fr, kevish.napalecmap. polytechnique.fr
} 
geneous media containing sound-hard cracks. GLSM provides an exact characterization of the target shape from the far field operator, and its implementation mainly requires two complementary factorizations of the far field operator, one used in the Linear Sampling Method (LSM) and another used in the Factorization Method (FM). From the measurements for both the damaged background and the initial background, it is possible to detect the defect thanks to the DLSM. This method consists in combining a result of comparison of two interior transmission problems associated with each background and the results of the GLSM.

The purpose of this article is to establish a similar factorization for a medium containing sound-hard cracks and to provide the theoretical results needed in the justification of the DLSM, the method we use to identify emergence of defects in an unknown background. For references of works dealing with qualitative methods to detect cracks, we mention, among others, $[5,6,11,4]$.

\section{The forward scattering problem}

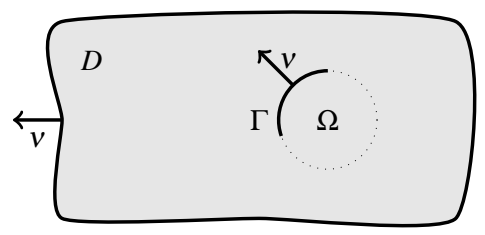

Fig. 1: Example of setting in $\mathbb{R}^{2}$.

We consider an isotropic medium embedded in $\mathbb{R}^{d}, d=2$ or 3 , containing soundhard cracks. Following [6], a crack $\Gamma$ is defined as a portion of a smooth nonintersecting curve $(d=2)$ or surface $(d=3)$ that encloses a domain $\Omega$, such that its boundary $\partial \Omega$ is smooth. We assume that $\Gamma$ is an open set with respect to the induced topology on $\partial \Omega$. The normal vector $v$ on $\Gamma$ is defined as the outward normal vector to $\Omega$ (see Fig. 1). To define traces and normal derivatives of functions on $\Gamma$, we use the following notation for all $x \in \Gamma$ :

$$
f^{ \pm}(x)=\lim _{h \rightarrow 0^{+}} f(x \pm h v(x)) \quad \text { and } \quad \partial_{v}^{ \pm} f(x)=\lim _{h \rightarrow 0^{+}} v(x) . \nabla f(x \pm h v(x)) .
$$

We shall also work with the jump functions

$$
[f]:=f^{+}-f^{-} \quad \text { and } \quad\left[\frac{\partial f}{\partial v}\right]:=\partial_{v}^{+} f-\partial_{v}^{-} f .
$$


We assume that the propagation of waves in time harmonic regime in the reference medium is governed by the Helmholtz equation $\Delta u+k^{2} u=0$ in $\mathbb{R}^{d}$ where $\Delta$ stands for the Laplace operator of $\mathbb{R}^{d}$ and where $k$ is the wave number. We assume that the cracks are embedded in a local perturbation of the reference medium. To model this perturbation, we introduce $n \in L^{\infty}\left(\mathbb{R}^{d}\right)$ a complex valued coefficient (the refractive index of the medium) such that $n=1$ in $\mathbb{R}^{d} \backslash \bar{D}$ and $n \neq 1$ in $D$. Here $D \subset \mathbb{R}^{d}$ is a bounded domain with Lipschitz boundary $\partial D$ such that $\mathbb{R}^{d} \backslash D$ is connected. We assume that $\mathfrak{I} m(n) \geq 0$ in $\mathbb{R}^{d}$ and that $\Gamma \subset D$. The scattering of the incident plane wave $u_{i}(\theta, \cdot):=e^{i k \theta \cdot x}$ of direction of propagation $\theta \in \mathbb{S}^{d-1}$ by the medium is described by the problem

$$
\mid \begin{aligned}
& \text { Find } u=u_{i}+u_{s} \text { such that } \\
& \Delta u+k^{2} n u=0 \text { in } \mathbb{R}^{d} \backslash \Gamma \\
& \quad \partial_{v}^{ \pm} u=0 \text { on } \Gamma \\
& \lim _{r \rightarrow+\infty} r^{\frac{d-1}{2}}\left(\frac{\partial u_{s}}{\partial r}-i k u_{s}\right)=0,
\end{aligned}
$$

with $u_{i}=u_{i}(\theta, \cdot)$. The last line of (1), where $r=|x|$, is the Sommerfeld radiation condition which selects the outgoing scattered field and which is assumed to hold uniformly with respect to $\widehat{x}=x /|x| \in \mathbb{S}^{d-1}$. For all $k>0$, Problem (1) has a unique solution $u$ belonging to $H^{1}(\mathscr{O} \backslash \Gamma)$ for all bounded domain $\mathscr{O} \subset \mathbb{R}^{d}$. The scattered field $u_{s}(\theta, \cdot)$ has the expansion

$$
u_{s}(\theta, x)=\eta_{d} e^{i k r} r^{-\frac{d-1}{2}}\left(u_{s}^{\infty}(\theta, \widehat{x})+O(1 / r)\right),
$$

as $r \rightarrow+\infty$, uniformly in $\widehat{x}=x /|x| \in \mathbb{S}^{d-1}$. In (2) the constant $\eta_{d}$ is given by $\eta_{d}=$ $e^{i \frac{\pi}{4}} / \sqrt{8 \pi k}$ for $d=2$ and by $=1 /(4 \pi)$ for $d=3$. The function $u_{s}^{\infty}(\theta, \cdot): \mathbb{S}^{d-1} \rightarrow \mathbb{C}$, is called the far field pattern associated with $u_{i}(\theta, \cdot)$. From the far field pattern, we can define the far field operator $F: L^{2}\left(\mathbb{S}^{d-1}\right) \rightarrow L^{2}\left(\mathbb{S}^{d-1}\right)$ such that

$$
(F g)(\widehat{x})=\int_{\mathbb{S}^{d-1}} g(\theta) u_{s}^{\infty}(\theta, \widehat{x}) d s(\theta) .
$$

By linearity, the function $\mathrm{Fg}$ corresponds to the far field pattern of the scattered field in (1) with

$$
u_{i}=v_{g}:=\int_{\mathbb{S}^{d-1}} g(\theta) e^{i k \theta \cdot x} d s(\theta) \quad \text { (Herglotz wave function). }
$$

\section{Factorization of the far field operator}

In this section we explain how to factorize the far field operator $F$ defined in (3). From the Green representation theorem, computing the asymptotic behaviour of the Green's function as $r \rightarrow+\infty$ gives 


$$
u_{s}^{\infty}(\widehat{x})=\left(k^{2} \int_{D}(n(y)-1) u(y) e^{-i k \widehat{x y}} d y+\int_{\Gamma}[u(y)] \partial_{v(y)}^{+} e^{-i k \hat{x} y} d s(y)\right)
$$

for the far field pattern of $u_{s}$ in (2). A first step towards the factorization of $F$ is to define the Herglotz operator $H: L^{2}\left(\mathbb{S}^{d-1}\right) \rightarrow L^{2}(D) \times L^{2}(\Gamma)$ such that

$$
H g=\left(v_{g_{\mid D}}, \partial_{v}^{+} v_{g \mid \Gamma}\right) .
$$

We give in Proposition 1 below a characterization of the closure of the range of $H$. Set

$$
\mathscr{H}=\left\{v \in L^{2}(D) \mid \Delta v+k^{2} v=0 \text { in } D\right\} .
$$

and define the map $\Psi: \mathscr{H} \rightarrow L^{2}(D) \times L^{2}(\Gamma)$ such that

$$
\Psi v=\left(v_{\mid D}, \partial_{v}^{+} v_{\mid \Gamma}\right) .
$$

Proposition 1. The operator $H: L^{2}\left(\mathbb{S}^{d-1}\right) \rightarrow L^{2}(D) \times L^{2}(\Gamma)$ defined in $(6)$ is injective and $\overline{R(H)}=\Psi(\mathscr{H})$.

Proof. The proof of the injectivity of $H$ follows a classical argument based on the Jacobi Anger expansion (apply [7, Lemma 2.1]). To establish the second part of the claim, first we note that $v_{g}$ (defined in (4)) belongs to $\mathscr{H}$ so that $R(H) \subset \Psi(\mathscr{H})$. On the other hand, classical results of interior regularity ensure that is some constant $C>0$ such that $\left\|\partial_{v} v\right\|_{L^{2}(\Gamma)} \leq C\|v\|_{L^{2}(D)}$ for all $v \in \mathscr{H}$. This in addition to $\|\Psi v\|_{L^{2}(D) \times L^{2}(\Gamma)} \geq\|v\|_{L^{2}(D)}$ allows one to show that $\Psi(\mathscr{H})$ is a closed subspace of $L^{2}(D) \times L^{2}(\Gamma)$. The regularity result implies that $\Psi:\left(\mathscr{H},\|\cdot\|_{L^{2}(D)}\right) \rightarrow$ $L^{2}(D) \times L^{2}(\Gamma)$ is continuous. Since the set of Herglotz wave functions is dense in $\left(\mathscr{H},\|\cdot\|_{L^{2}(D)}\right)$, we deduce that $\overline{R(H)}=\Psi(\mathscr{H})$.

Next we define the operator $G: \overline{R(H)} \rightarrow L^{2}\left(\mathbb{S}^{d-1}\right)$ such that

$$
G\left(v, \partial_{v}^{+} v\right)=u_{s}^{\infty}
$$

where $u_{s}^{\infty}$ is the far field pattern of $u_{s}$, the outgoing scattered field which satisfies

$$
\mid \begin{aligned}
\Delta u_{s}+k^{2} n u_{s} & =k^{2}(1-n) v & & \text { in } \mathbb{R}^{d} \backslash \Gamma \\
\partial_{v}^{ \pm} u_{s} & =-\partial_{v}^{ \pm} v & & \text { on } \Gamma .
\end{aligned}
$$

Note that if $\left(v, \partial_{v}^{+} v\right) \in \overline{R(H)}$ then interior regularity implies $\partial_{v}^{+} v=\partial_{v}^{-} v$ on $\Gamma$. We also define the map $T: L^{2}(D) \times L^{2}(\Gamma) \rightarrow L^{2}(D) \times L^{2}(\Gamma)$ such that

$$
T\left(v, \partial_{v}^{+} v\right)=\left(k^{2}(n-1)\left(v+u_{s}\right),\left[v+u_{s}\right]\right) .
$$

Clearly we have $F=G H$. And one can check using (5) that $G=H^{*} T$ so that $F$ admits the factorisation

$$
F=H^{*} T H \text {. }
$$


The justification of the techniques we propose below to recover the cracks will depend on the properties of the operators $G, T$. And the latter are related to the solvability of the so-called interior transmission problem which in our situation states as follows: given $f \in H^{3 / 2}(\partial D), g \in H^{1 / 2}(\partial D)$

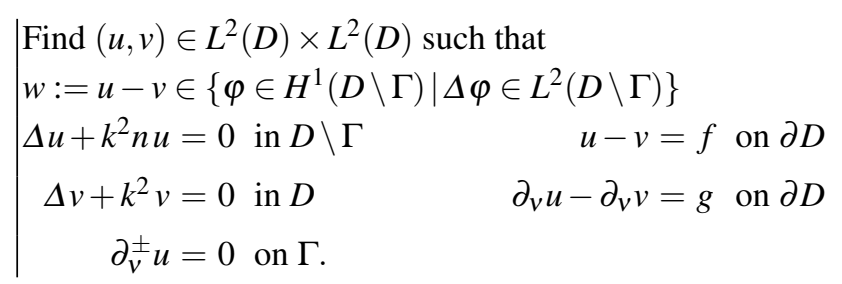

We shall say that $k>0$ is a transmission eigenvalue if (13) with $f=g=0$ admits a non zero solution. One can show for example that if the coefficient $n$ is real and satisfies $1<n_{*}<n<n^{*}$ for some constants $n_{*}, n^{*}$, then the set of transmission eigenvalues is discrete without accumulation point and that Problem (13) is uniquely solvable if and only if $k$ is not a transmission eigenvalue (this will be part of a future work). We shall say that (13) is well-posed if it admits a unique solution for all $f \in H^{3 / 2}(\partial D), g \in H^{1 / 2}(\partial D)$.

Proposition 2. Assume that $k>0$ is not a transmission eigenvalue. Then the operator $G: \overline{R(H)} \rightarrow L^{2}\left(\mathbb{S}^{d-1}\right)$ is compact, injective with dense range.

Proof. First we show the injectivity of $G$. Let $V=\left(v, \partial_{v}^{+} v\right) \in \overline{R(H)}$ such that $G V=$ 0 . Then from the Rellich lemma, the solution $u_{s}$ of (10) is zero in $\mathbb{R}^{d} \backslash \bar{D}$. Therefore, if we define $u=v+u_{s}$, then the pair $(u, v)$ satisfies the interior transmission problem (13) with $f=g=0$. Since we assumed that $k>0$ is not a transmission eigenvalue, we deduce that $v=0$ and so $V=0$.

Now we focus our attention on the denseness of the range of $G$. First we establish an identity of symmetry. Let $V_{1}=\left(v_{1}, \partial_{v}^{+} v_{1}\right), V_{2}=\left(v_{2}, \partial_{v}^{+} v_{2}\right) \in \overline{R(H)}$. Denote $w_{1}$, $w_{2}$ the corresponding solutions to Problem (10). In particular we have

$$
\Delta w_{1}+k^{2} n w_{1}=k^{2}(1-n) v_{1}, \quad \Delta w_{2}+k^{2} n w_{2}=k^{2}(1-n) v_{2} \quad \text { in } \mathbb{R}^{d} \backslash \Gamma .
$$

Multiplying the first equation by $w_{2}$ and the second by $w_{1}$, integrating by parts the difference over $B_{R}$, the open ball of radius $R$ centered at $O$, we obtain

$$
\begin{aligned}
& k^{2} \int_{D}(n-1)\left(v_{1} w_{2}-v_{2} w_{1}\right) d x \\
&=\int_{\partial B_{R}}\left(\partial_{v} w_{1} w_{2}-w_{1} \partial_{v} w_{2}\right) d s+\int_{\Gamma}\left(\left[w_{2}\right] \partial_{v}^{+} v_{1}-\left[w_{1}\right] \partial_{v}^{+} v_{2}\right) d s .
\end{aligned}
$$

Taking the limit as $R \rightarrow+\infty$ and using that $\lim _{R \rightarrow+\infty} \int_{\partial B_{R}}\left(\partial_{\nu} w_{1} w_{2}-w_{1} \partial_{\nu} w_{2}\right) d s=0$ ( $w_{1}$ and $w_{2}$ satisfy the radiation condition), we find the identity

$$
k^{2} \int_{D}(n-1) v_{1} w_{2} d x+\int_{\Gamma} \partial_{v}^{+} v_{1}\left[w_{2}\right] d s=k^{2} \int_{D}(n-1) v_{2} w_{1} d x+\int_{\Gamma} \partial_{v}^{+} v_{2}\left[w_{1}\right] d s .
$$


Using (15), we deduce that for $\phi, g \in L^{2}\left(\mathbb{S}^{d-1}\right)$, we have

$$
\begin{aligned}
& \langle G(H \phi), \bar{g}\rangle_{L^{2}\left(\mathbb{S}^{d-1}\right)} \\
= & k^{2} \int_{D}(n-1)\left(H \phi+u_{s}(\phi)\right) H g d x+\int_{\Gamma}\left[H \phi+u_{s}(\phi)\right] \partial_{v}^{+}(H g) d s \\
= & k^{2} \int_{D}(n-1)\left(H g+u_{S}(g)\right) H \phi d x+\int_{\Gamma}\left[H g+u_{s}(g)\right] \partial_{v}^{+}(H \phi) d s \\
= & \langle G(H g), \bar{\phi}\rangle_{L^{2}\left(\mathbb{S}^{d-1}\right)} .
\end{aligned}
$$

Therefore if $\bar{g} \in R(G)^{\perp}$ then $G(H g)=0$. The injectivity of $G$ and $H$ imply that $g=0$ which shows that $G$ has dense range.

Finally, using again the estimate $\left\|\partial_{v} v\right\|_{L^{2}(\Gamma)} \leq C\|v\|_{L^{2}(D)}$ for all $v \in \mathscr{H}$, results of interior regularity and the definition of $H$ (see (6)), one can check that $H: L^{2}\left(\mathbb{S}^{d-1}\right) \rightarrow$ $L^{2}(D) \times L^{2}(\Gamma)$ is compact. Since $G=H^{*} T$ and $T$ is continuous, we deduce that $G: L^{2}(D) \times L^{2}(\Gamma) \rightarrow L^{2}\left(\mathbb{S}^{d-1}\right)$ is compact.

Proposition 3. For all $V=\left(v, \partial_{v}^{+} v\right) \in \overline{R(H)}$, we have the energy identity

$$
\mathfrak{I} m\left(\langle T V, V\rangle_{L^{2}(D) \times L^{2}(\Gamma)}\right)=k^{2} \int_{D} \mathfrak{I} m(n)\left|u_{s}+v\right|^{2} d x+k\|G V\|_{L^{2}\left(\mathbb{S}^{d-1}\right)}^{2},
$$

where $u_{s}$ denotes the solution of (10). As a consequence if $\Im m(n) \geq 0$ a.e. in $D$ and if $k$ is not a transmission eigenvalue of (13), then $T$ is injective.

Proof. Multiplying by $\overline{u_{s}}$ the equation $\Delta u_{s}+k^{2} u_{s}=-k^{2}(n-1)\left(u_{s}+v\right)$ and integrating by parts over the ball $B_{R}$, we obtain

$$
\begin{aligned}
-k^{2} \int_{D}(n-1) & \left(u_{s}+v\right) \overline{u_{s}} d x= \\
& -\int_{B_{R}}\left|\nabla u_{s}\right|^{2}-k^{2}\left|u_{s}\right|^{2} d x+\int_{\partial B_{R}} \partial_{v} u_{s} \overline{u_{S}} d s-\int_{\Gamma} \partial_{v}^{+} u_{S}\left[\overline{u_{s}}\right] d s .
\end{aligned}
$$

Using (17), then we find

$$
\begin{aligned}
\langle T V, V\rangle_{L^{2}(D) \times L^{2}(\Gamma)} & =k^{2} \int_{D}(n-1)\left|u_{s}+v\right|^{2} d x-\int_{B_{R}}\left|\nabla u_{S}\right|^{2}-k^{2}\left|u_{s}\right|^{2} d x \\
& +\int_{\Gamma}\left[v+u_{S}\right] \partial_{v}^{+} \bar{v} d s-\int_{\Gamma} \partial_{v}^{+} u_{s}\left[\overline{u_{S}}\right] d s+\int_{\partial B_{R}} \partial_{v} u_{s} \overline{u_{S}} d s .
\end{aligned}
$$

Since $\partial_{v}^{+} u_{s}=-\partial_{v}^{+} v$ and $[v]=0$ (interior regularity) on $\Gamma$, we deduce

$$
\begin{aligned}
\langle T V, V\rangle_{L^{2}(D) \times L^{2}(\Gamma)=} & k^{2} \int_{D}(n-1)\left|u_{s}+v\right|^{2} d x-\int_{B_{R}}\left|\nabla u_{s}\right|^{2}-k^{2}\left|u_{s}\right|^{2} d x \\
& -2 \Re e\left(\int_{\Gamma}\left[u_{s}\right] \partial_{v}^{+} \overline{u_{s}} d s\right)+\int_{\partial B_{R}} \partial_{v} u_{s} \overline{u_{s}} d s .
\end{aligned}
$$

The radiation condition (see (1)) implies $\lim _{R \rightarrow \infty} \int_{\partial B_{R}} \partial_{v} u_{s} \overline{u_{s}} d s=i k \int_{\mathbb{S}^{d-1}}\left|u_{s}^{\infty}\right|^{2} d \theta=$ $i k\|G V\|_{L^{2}\left(\mathbb{S}^{d-1}\right)}^{2}$. As a consequence, taking the imaginary part of (18) and letting $R$ 
goes to infinity, we get identity (16). Now if $T V=0$ and if $\Im m(n) \geq 0$ a.e. in $D$, then (16) gives $G V=0$. Since $G$ is injective when $k$ is not a transmission eigenvalue of (13) (Proposition 2), we deduce that $T$ is injective.

\section{Generalized Linear Sampling Method and Differential Linear Sampling Method}

For $z \in \mathbb{R}^{d}$, we denote by $\Phi(., z)$ the outgoing fundamental solution of the homogeneous Helmoltz equation such that

$$
\Phi(x, z)=\frac{i}{4} H_{0}^{(1)}(k|x-z|) \text { if } d=2 \quad \text { and } \quad \frac{e^{i k|x-z|}}{4 \pi|x-z|} \text { if } d=3 .
$$

Here $H_{0}^{(1)}$ stands for the Hankel function of first kind of order zero. The far field of $\Phi(., z)$ is $\phi_{z}(\widehat{x})=e^{-i k z . \widehat{x}}$. The GLSM uses the following theorem whose proof is classical [7].

Theorem 1. Assume that the interior transmission problem (13) is well-posed. Then

$$
z \in D \quad \text { if and only if } \quad \phi_{z} \in R(G) .
$$

The particularity of the GLSM is to build an approximate solution $\left(F g \simeq \phi_{z}\right)$ to the far field equation by minimizing the functional $J^{\alpha}\left(\phi_{z},.\right): L^{2}\left(\mathbb{S}^{d-1}\right) \rightarrow \mathbb{R}$ defined by

$$
J^{\alpha}\left(\phi_{z}, g\right)=\alpha\left\langle F^{\sharp} g, g\right\rangle_{L^{2}\left(\mathbb{S}^{d-1}\right)}+\left\|F g-\phi_{z}\right\|_{L^{2}\left(\mathbb{S}^{d-1}\right)}^{2}, \quad \forall g \in L^{2}\left(\mathbb{S}^{d-1}\right),
$$

where $F^{\sharp}:=\left|\frac{1}{2}\left(F+F^{*}\right)\right|+\left|\frac{1}{2 i}\left(F-F^{*}\right)\right|$.

Theorem 2 (GLSM). Assume that the interior transmission problem (13) is wellposed, that the index $n$ satisfies $\left[\Im m(n) \geq 0, \Re e(n-1) \geq n_{*}\right.$ a.e. in $\left.D\right]$ or $[\Im m(n) \geq$ $0, \Re e(1-n) \geq n_{*}$ a.e. in $\left.D\right]$ for some constant $n_{*}>0$. Let $g_{z}^{\alpha} \in L^{2}\left(\mathbb{S}^{d-1}\right)$ be a minimizing sequence of $J^{\alpha}\left(\phi_{z},.\right)$ such that

$$
J^{\alpha}\left(\phi_{z}, g_{z}^{\alpha}\right) \leq \inf _{g} J^{\alpha}\left(\phi_{z}, g\right)+p(\alpha),
$$

where $\lim _{\alpha \rightarrow 0} \alpha^{-1} p(\alpha)=0$. Then

- $z \in D \quad$ if and only if $\quad \lim _{\alpha \rightarrow 0}\left\langle F^{\sharp} g_{z}^{\alpha}, g_{z}^{\alpha}\right\rangle_{L^{2}\left(\mathbb{S}^{d-1}\right)}<+\infty$.

- If $z \in D$ then there exists $h \in \overline{R(H)}$ such that $\phi_{z}=G h$ and $H g_{z}^{\alpha}$ converges strongly to $h$ as $\alpha \rightarrow 0$.

Thus the GLSM, justified by this theorem, offers a way to recover $D$, that is to identify the perturbation in the reference background. Note that the GLSM, contrary to the LSM, provides an exact characterization of $D$. However it does not give any information on the location of the $\operatorname{crack} \Gamma$. 
Proof. We establish this theorem by applying the abstract result of [7, Theorem 2.10]. The latter requires that the following properties hold.

i) $F=G H=H^{*} T H$ is injective with dense range and $G$ is compact.

ii) $F^{\sharp}$ factorizes as $F^{\sharp}=H^{*} T^{\sharp} H$ where $T^{\sharp}$ satisfies the coercivity property

$$
\exists \mu>0, \forall V \in \overline{R(H)}, \quad\left|\left\langle T^{\sharp} V, V\right\rangle_{L^{2}(D) \times L^{2}(\Gamma)}\right| \geq \mu\|V\|_{L^{2}(D) \times L^{2}(\Gamma)}^{2} ;
$$

iii) $V \mapsto\left|\left\langle T^{\sharp} V, V\right\rangle_{L^{2}(D) \times L^{2}(\Gamma)}\right|^{1 / 2}$ is uniformly convex on $\overline{R(H)}$.

Item $i$ ) is a consequence of Propositions 1, 2 and 3. Moreover, we deduce iii) from ii) and from the fact that $\left\langle F^{\sharp} g, g\right\rangle_{L^{2}(D) \times L^{2}(\Gamma)}=\left\|\left(F^{\sharp}\right)^{1 / 2} g\right\|_{L^{2}\left(\mathbb{S}^{d-1}\right)}^{2}$ (see e.g. [7]). Therefore, it remains to show $i i)$. To proceed, we use [7, Theorem 2.31] which guarantees that it is true if :

- $T$ injective on $\overline{R(H)}$;

- $\mathfrak{I} m\left(\langle T V, V\rangle_{L^{2}(D) \times L^{2}(\Gamma)}\right) \geq 0$ for all $V \in \overline{R(H)}$;

- $\mathfrak{R} e(T)$ decomposes as $\mathfrak{R} e(T)=T_{0}+C$ where $T_{0}$ satisfies (22) and where $C$ is compact on $\overline{R(H)}$.

The first two items have been proved in Proposition 3. Let us focus our attention on the last one. By definition, we have $T V=\left(k^{2}(n-1)\left(v+u_{s}\right),\left[v+u_{s}\right]\right)$. Set $\tilde{C} V=$ $\left(k^{2}(n-1) u_{s},\left[v+u_{s}\right]-\left.\partial_{v}^{+} v\right|_{\Gamma}\right)$. Using results of interior regularity, one can check that $C=\Re e(\tilde{C})$ is compact. Now, define $T_{0}:=\Re e(T)-C=\left(k^{2} \Re e(n-1) v,\left.\partial_{v}^{+} v\right|_{\Gamma}\right)$. Clearly one has $\left|\left\langle T_{0} V, V\right\rangle_{L^{2}(D) \times L^{2}(\Gamma)}\right| \geq n_{*}\|V\|_{L^{2}(D) \times L^{2}(\Gamma)}^{2}$ when $\Re e(n-1) \geq n_{*}$. The case $\mathfrak{R} e(1-n) \geq n_{*}$ can be dealt in a similar way.

When one has only acces to a noisy version $F^{\delta}$ of $F$, then $F^{\sharp, \delta}$ might not have the required factorization and the cost function (21) must be regularized. For this aspect, we refer the reader to [2, Section 5.2].

We now give the theoretical foundation of the DLSM which will allow us to localize the position of the crack $\Gamma$. The DLSM relies on the comparison of the solutions of the following interior transmission problems (without and with cracks).

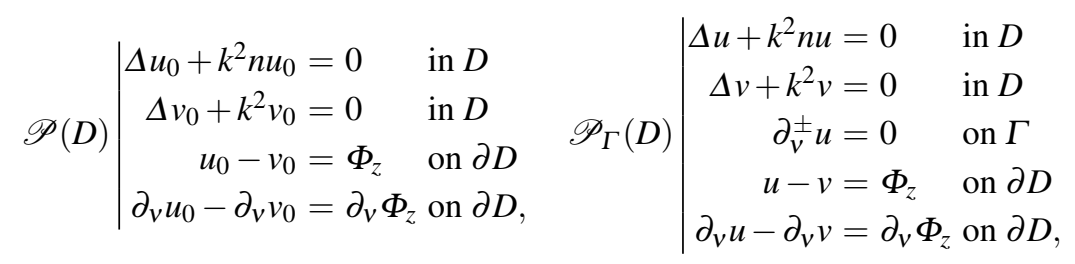

where $u_{0}, v_{0}, u, v \in L^{2}(D), u_{0}-v_{0} \in H^{2}(D)$ and $u-v \in H^{1}(D \backslash \Gamma)$ is such that $\Delta(u-$ $v) \in L^{2}(D \backslash \Gamma)$. We split the domain $D$ into two kinds of connected components (see Fig. 2): The ones containing cracks are listed by $\left(D_{\Gamma}^{j}\right)_{j}$; others are listed by $\left(D_{0}^{j}\right)_{j}$. And we set $D_{\Gamma}:=\cup_{j} D_{\Gamma}^{j}$ and $D_{0}:=\cup_{j} D_{0}^{j}$ so that $D=D_{\Gamma} \cup D_{0}$.

Theorem 3. Assume that $\Gamma$ is a part of the boundary of a domain $\Omega$ such that $\partial \Omega$ is analytic. Assume that $n$ is analytic in $D_{\Gamma}$ and does not vanish. Assume also that 


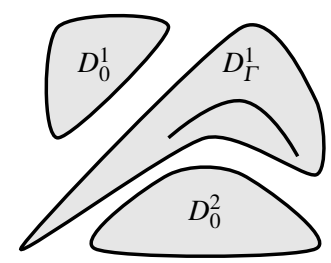

Fig. 2: We split $D$ into two families of connected components.

$k$ is not a Neumann eigenvalue for $-n^{-1} \Delta$ in $\Omega$ and is such that both $\mathscr{P}(D)$ and $\mathscr{P}_{\Gamma}(D)$ (see (23)) are well-posed.
i) If $z \in D_{0}$ then $v=v_{0}$ in $D$.
ii) If $z \in D_{\Gamma}$ then $v \neq v_{0}$ in $D_{\Gamma}$.

Proof. i) Let $z \in D_{0}$. In $D_{0}$, the equations for $\mathscr{P}(D)$ and $\mathscr{P}_{\Gamma}(D)$ coincide. By uniqueness of the solution for these problems, we deduce that $v=v_{0}$ in $D_{0}$. On the other hand, one observes that $\left(0,-\Phi_{z}\right)$ satisfies the equations of $\mathscr{P}(D)$ and $\mathscr{P}_{\Gamma}(D)$ in $D_{\Gamma}$. As a consequence, by uniqueness of the solution for these problems, we also have $v=v_{0}=-\Phi_{z}$ in $D_{\Gamma}$.

ii) Now let $z \in D_{\Gamma}$. We wish to show that $v \neq v_{0}$ in $D_{\Gamma}$. We proceed by contradiction assuming that $v=v_{0}$ in $D_{\Gamma}$. Define $U$ such that $U=u-u_{0}$ in $D_{\Gamma} \backslash \Gamma$ and $U=0$ in $\mathbb{R}^{d} \backslash D_{\Gamma}$. Since $U=\partial_{\nu} U=0$ on $\partial D_{\Gamma}$, from the unique continuation principle, we find $U=0$ in $\mathbb{R}^{d} \backslash \Gamma$ and so $\partial_{v}^{ \pm} u_{0}=0$ on $\Gamma$ (because $\partial_{v}^{ \pm} u=0$ on $\Gamma$ ). Furthermore the regularity of $n$ implies that $\partial_{v}^{ \pm} u_{0}$ is analytic on $\partial \Omega$ and we conclude that $\partial_{v}^{ \pm} u_{0}=0$ on $\partial \Omega$. Since we assumed that $k$ is not a Neumann eigenvalue for $-n^{-1} \Delta$ in $\Omega$, we deduce that $u_{0}=0$ in $\Omega$, and by unique continuation, $u_{0}=0$ in $D_{\Gamma}$. Thus we must have $v_{0}=-\Phi_{z}$ in $D_{\Gamma}$ which contradicts the fact that $u_{0}-v_{0} \in H^{2}(D)$.

Now we consider a first heterogeneous medium without crack with a perturbation of the reference background supported in $\bar{D}$ modeled by some index $n$, and a second medium with the same $n$ but with an additional crack inside $D$. The corresponding far field operators are denoted respectively $F_{0}$ and $F_{1}$. Then for $j=0,1$, let $g_{j, z}^{\alpha}$ refer to the sequences introduced in the statement of Theorem 2 with $F_{j}^{\sharp}=\left|\frac{1}{2}\left(F_{j}+F_{j}^{*}\right)\right|+$ $\left|\frac{1}{2 i}\left(F_{j}-F_{j}^{*}\right)\right|$. We also set for $j=0,1$

$$
\mathscr{A}_{j}^{\alpha}(z)=\left\langle F_{j}^{\sharp} g_{j, z}^{\alpha}, g_{j, z}^{\alpha}\right\rangle_{L^{2}\left(\mathbb{S}^{d-1}\right)} ; \mathscr{D}_{j}^{\alpha}(z)=\left\langle F_{j}^{\sharp}\left(g_{1, z}^{\alpha}-g_{0, z}^{\alpha}\right),\left(g_{1, z}^{\alpha}-g_{0, z}^{\alpha}\right)\right\rangle_{L^{2}\left(\mathbb{S}^{d-1}\right)} .
$$

The combination of Theorem 2 and 3 leads to the following result.

Theorem 4 (DLSM). Assume that $k, n$ and $\Gamma$ are as in Theorem 3 and that $n$ also satisfies the assumptions of Theorem 2. Then for $j=0$ or 1

$$
\left[z \in D_{0}\right] \Rightarrow\left[\lim _{\alpha \rightarrow 0} \mathscr{D}_{j}^{\alpha}(z)=0\right] \quad \text { and } \quad\left[z \in D_{\Gamma}\right] \Rightarrow\left[0<\lim _{\alpha \rightarrow 0} \mathscr{D}_{j}^{\alpha}(z)<+\infty\right] \text {. }
$$

Proof. As explained in the proof of Theorem 2, $F_{1}^{\sharp}$ admits a factorization of the form $H^{*} T_{1}^{\sharp} H$ where $T_{1}^{\sharp}$ is continuous and $\left\langle T_{1}^{\sharp}, \cdot\right\rangle$ is coercive. According to the study 
of crack-free inhomogeneous medium a same factorization stands for $F_{0}$ involving an operator $T_{0}^{\sharp}$ that have the same properties of $T_{1}^{\sharp}$. This implies (for $j=0$ or 1 ) the existence of two positive constants $\kappa$ and $K$ such that

$$
\kappa\left\|H\left(g_{1, z}^{\alpha}-g_{0, z}^{\alpha}\right)\right\|_{L^{2}(D)}^{2} \leq \mathscr{D}_{j}^{\alpha}(z) \leq K\left\|H\left(g_{1, z}^{\alpha}-g_{0, z}^{\alpha}\right)\right\|_{L^{2}(D)}^{2} .
$$

Now for $z \in D$, if we denote $\left(u_{0}, v_{0}\right)$ (resp. $\left.\left(u_{1}, v_{1}\right)\right)$ the solution of $\mathscr{P}(D)$ (resp. $\mathscr{P}_{\Gamma}(D)$ ), then Theorem 2 and the GLSM for the crack-free inhomogeneous medium (see the justification in [7]) guarantee that $\lim _{\alpha \rightarrow 0}\left\|H\left(g_{1, z}^{\alpha}-g_{0, z}^{\alpha}\right)\right\|=\left\|H\left(v-v_{0}\right)\right\|$. Then the result follows from Theorem 3 .

From Theorems 2 and 4, one can design indicators for $D$ and $D_{\Gamma}$. Set for $j=0$ or 1 ,

$$
I^{\mathrm{GLSM}}(z)=\lim _{\alpha \rightarrow 0} \frac{1}{\mathscr{A}_{1}^{\alpha}(z)} \quad \text { and } \quad I_{j}^{\mathrm{DLSM}}(z)=\lim _{\alpha \rightarrow 0} \frac{1}{\mathscr{A}_{0}^{\alpha}(z)\left(1+\frac{\mathscr{A}_{0}^{\alpha}(z)}{\mathscr{D}_{j}^{\alpha}(z)}\right)} .
$$

For these indicators, one can show the following theorem which allows one to identify the connected components of $D$ in which some cracks have appeared.

Corollary 1. Under the assumptions of Theorem 4, we have for $j=0$ or 1

$$
\begin{aligned}
& \text { - } I^{\mathrm{GLSM}}(z)=0 \text { in } \mathbb{R}^{d} \backslash D \quad \text { and } \quad I^{\mathrm{GLSM}}(z)>0 \text { in } D . \\
& \text { - } I_{j}^{\mathrm{DLSM}}(z)=0 \text { in } \mathbb{R}^{d} \backslash D_{\Gamma} \quad \text { and } \quad I_{j}^{\mathrm{DLSM}}(z)>0 \text { in } D_{\Gamma} .
\end{aligned}
$$

\section{Numerical results}

To conclude this work, we apply the GLSM and the DLSM on simulated backgrounds. All backgrounds have the same shape $D$ constituted of three disjoint disks of radius 0.75 and of index $n=1.5$. They differ from one to another in the distribution of cracks inside the disks. Admittedly, the straight cracks appearing in the backgrounds are not a portion of the boundary of an analytic domain. However, we expect that our algorithm remains robust when this theoretical assumption is not satisfied. For each background we generate a discretization of the far field operator $F$ by solving numerically the direct problem for multiple incident fields $u_{i}\left(\theta_{p}\right)$ with wave number $k=4 \pi$. Then we compute the matrix $F=\left(u_{s}^{\infty}\left(\theta_{p}, \widehat{x_{q}}\right)\right)_{p, q}$ for $\theta_{p}, \widehat{x_{q}}$ in $\left\{\cos \left(\frac{2 l \pi}{100}\right), \sin \left(\frac{2 l \pi}{100}\right), l=1 . .100\right\}$ (somehow we discretize $L^{2}\left(\mathbb{S}^{1}\right)$ ). Finally, we add random noise to the simulated $F$ and obtain our final synthetic far field data $F^{\delta}$ with $F_{p q}^{\delta}=F_{p q}(1+\sigma N)$. Here $N$ is a complex random variable whose real and imaginary parts are uniformly chosen in $[-1,1]^{2}$. The parameter $\sigma>0$ is chosen so that $\left\|F^{\delta}-F\right\|=0.05\left\|F^{\delta}\right\|$. 


\subsection{GLSM}

To handle the noise $\delta$ added on the far field data, we use a regularized version of the GLSM consisting in finding the minimizers $g_{z}^{\alpha, \delta}$ of the functional

$$
g \mapsto J^{\alpha, \delta}\left(\phi_{z}, g\right)=\alpha\left(\left|\left\langle F^{\sharp \delta} g, g\right\rangle_{L^{2}\left(\mathbb{S}^{d-1}\right)}\right|+\delta\left\|F^{\delta}\right\|\|g\|_{L^{2}\left(\mathbb{S}^{2}\right)}^{2}\right)+\left\|F^{\delta} g-\phi_{z}\right\|_{L^{2}\left(\mathbb{S}^{d-1}\right)}^{2},
$$

where $F^{\sharp \delta}:=\left|\frac{1}{2}\left(F^{\delta}+F^{\delta *}\right)\right|+\left|\frac{1}{2 i}\left(F^{\delta}-F^{\delta *}\right)\right|$. We fit $\alpha$ to $\delta$ according to [2, Section 5.2]. The new relevant indicator function for the regularized GLSM is then given by

$$
I_{\mathrm{GLSM}}^{\alpha, \delta}(z)=\frac{1}{\mathscr{A}^{\alpha, \delta}(z)}
$$

where $\mathscr{A}^{\alpha, \delta}(z)=\left\langle F^{\sharp \delta} g_{z}^{\alpha, \delta}, g_{z}^{\alpha, \delta}\right\rangle_{L^{2}\left(\mathbb{S}^{d-1}\right)}+\delta\left\|F^{\delta}\right\|\left\|g_{z}^{\alpha, \delta}\right\|_{L^{2}\left(\mathbb{S}^{d-1}\right)}$.

Fig. 3 shows the results of GLSM indicator function $z \mapsto I_{\mathrm{GLSM}}^{\alpha, \delta}(z)$ for two different configurations where the second one is obtained from the first one by adding a crack to the third component. The two other components contain the same crack. One observes that GLSM is capable of retrieving the domain $D$ for each configuration. We also observe how the behavior of the indicator function is different inside the third component. This is somehow what the DLSM exploits to isolate the component where a defect appears and this is what is discussed next.
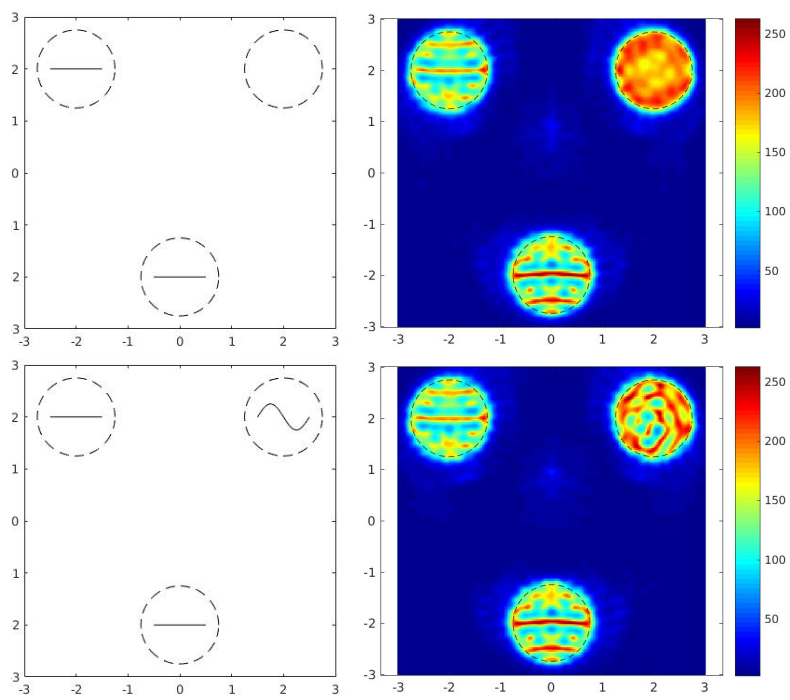

Fig. 3: Simulated backgrounds on the left and associated GLSM indicator function $z \mapsto I_{\mathrm{GLSM}}^{\alpha, \delta}(z)$ on the right. 


\subsection{DLSM}

Given two far field data $F_{0}^{\delta}$ and $F_{1}^{\delta}$, we respectively define $F_{0}^{\sharp \delta}, g_{0, z}^{\alpha, \delta}, \mathscr{A}_{0}^{\alpha, \delta}(z)$ and $F_{1}^{\sharp \delta}, g_{1, z}^{\alpha, \delta}, \mathscr{A}_{1}^{\alpha, \delta}(z)$ associated to each data as described in the previous paragraph. We also define

$$
\mathscr{D}^{\alpha, \delta}(z)=\left\langle F_{0}^{\sharp}\left(g_{1, z}^{\alpha, \delta}-g_{0, z}^{\alpha, \delta}\right),\left(g_{1, z}^{\alpha, \delta}-g_{0, z}^{\alpha, \delta}\right)\right\rangle_{L^{2}\left(\mathbb{S}^{d-1}\right)} .
$$

Then, according to (26), the DLSM indicator is given by

$$
I_{\mathrm{DLSM}}^{\alpha, \delta}(z)=\frac{1}{\mathscr{A}_{0}^{\alpha, \delta}(z)\left(1+\frac{\mathscr{A}_{0}^{\alpha, \delta}(z)}{\mathscr{D}^{\alpha, \delta}(z)}\right)} .
$$

The behavior of the DLSM indicator function is illustrated below for several scenarios shown in Fig. 4-7. In each figure is presented from left to right, the initial background (associated with $F_{0}^{\delta}$ ), the damaged background (associated with $F_{1}^{\delta}$ ) and the DLSM indicator function $z \mapsto I_{\mathrm{DLSM}}^{\alpha, \delta}(z)$. As expected, the latter allows us to identify for all scenarios the component(s) $D_{\Gamma}$ where (additional) cracks appeared. We also remark that it slightly accentuates the border of $D_{0}$. But this effect is not explained by our theory and it does not contradict it: Our theoretical result does not stipulate that the indicator function is "uniformly" close to 0 outside $D_{\Gamma}$.
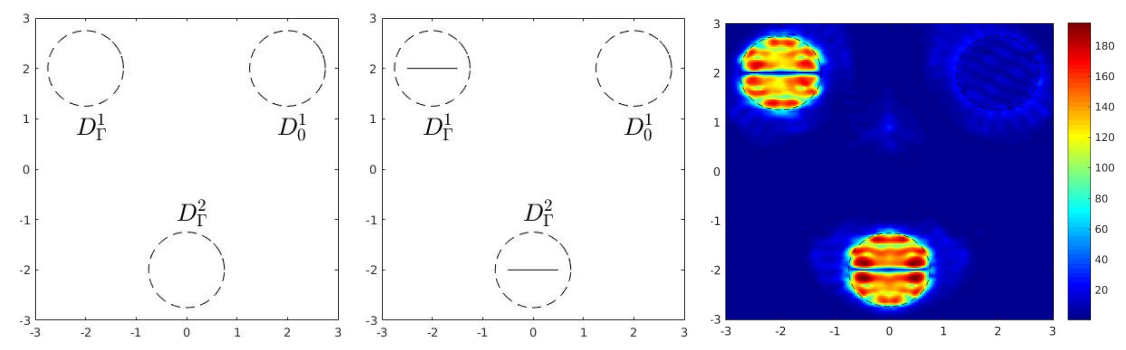

Fig. 4: A scenario for DLSM simulating the emergence of cracks in two components of a defect free background. 

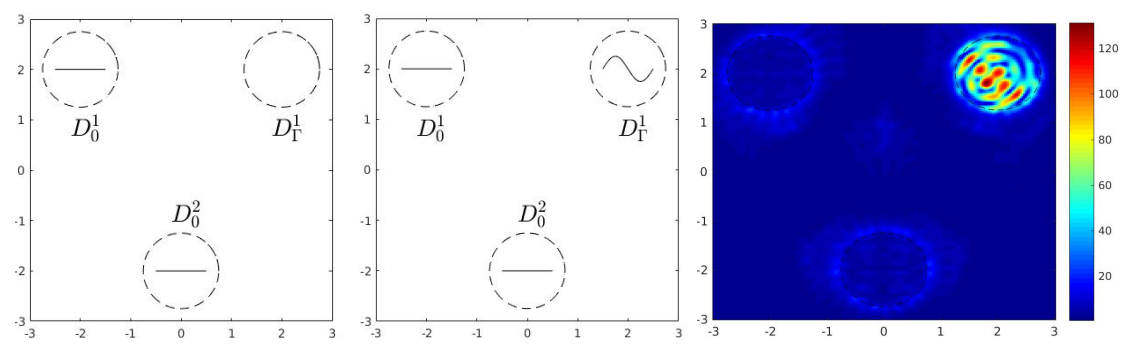

Fig. 5: A scenario for DLSM simulating the emergence of a crack in a healthy component of an already damaged background.
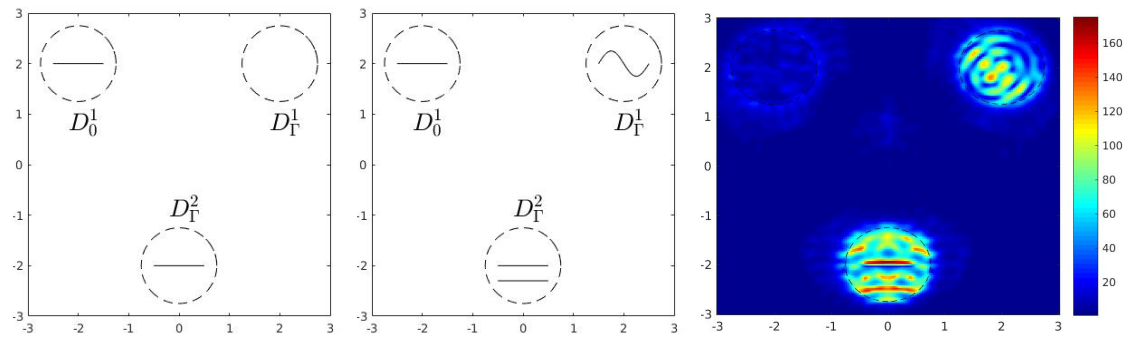

Fig. 6: A scenario for DLSM simulating the emergence of additional cracks in a healthy and a damaged components of an already damaged background.
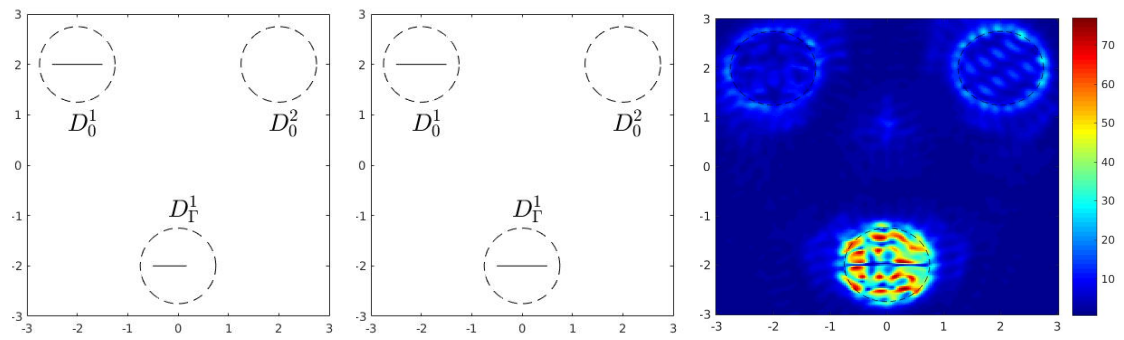

Fig. 7: A scenario for DLSM simulating the increase of the crack size in one component of an already damaged background.

\section{Conclusion}

We analyzed the DLSM to identify emergence of cracks embedded in an unknown background and image defective components from differential measurements of far 
field data at a fixed frequency. The analysis is based on the justification of the GLSM for backgrounds with cracks which necessitates the study of a special interior transmission problem and the derivation of specific factorizations of the far field operator. The numerical tests on toy problems show that our method is reliable for different scenarios simulating the appearance of cracks between two measurements

campaigns. This is a first step before addressing practical problems where the issues of limited aperture data and/or highly cluttered backgrounds should be solved.

\section{References}

1. L. Audibert, Qualitative methods for heterogeneous media. $\mathrm{PhD}$ thesis, École Doctorale Polytechnique, 2015.

2. L. Audibert, H. Haddar, A generalized formulation of the linear sampling method with exact characterization of targets in terms of farfeld measurements. Inverse Problems, 30 (035011), 2014.

3. L. Audibert, A. Girard, H. Haddar, Identifying defects in an unknown background using differential measurements. Inverse Problems \& Imaging, 9 (3) : 625-643, 2015.

4. F. Ben Hassen, Y. Boukari, H. Haddar, Application of the linear sampling method to retrieve cracks with impedance boundary conditions, Rapport de recherche RR-7478, INRIA, 2010.

5. L. Bourgeois, E. Lunéville, On the use of the Linear Sampling Method to identify cracks in elastic waveguides. Inverse Problems, 29 025017, 2013.

6. F. Cakoni, D. Colton, Qualitative methods in inverse scattering theory Interaction of Mechanics and Mathematics: An Introduction, Springer, 2006.

7. F. Cakoni, D. Colton, H. Haddar, Inverse Scattering Theory and Transmission Eigenvalues. Series in Applied Mathematics, 2016.

8. D. Colton, R. Kress, Inverse Acoustic and Electromagnetic Scattering Theory. SpringerVerlag, 1992.

9. D. Colton, R. Kress, Integral Equation Methods in Scattering Theory. John Wiley, 1993.

10. A. Kirsch, An Introduction to the Mathematical Theory of Inverse Problems. Springer, 2011.

11. A. Kirsch, S. Ritter A linear sampling method for inverse scattering from an open arc, Inverse Problems 16 89, 2000.

12. W. McLean, Strongly Ellyptic Systems and Boundary Integral Equations. Cambridge University Press, 2000. 